\title{
No. $2005-80$ \\ THE TWO-STAGE CONSTRAINED EQUAL AWARDS AND LOSSES RULES FOR MULTI-ISSUE ALLOCATION SITUATIONS
}

By Silvia Lorenzo-Freire, Balbina Casas-Méndez, Ruud Hendrickx

June 2005

ISSN 0924-7815

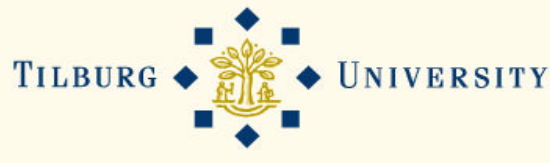




\title{
The two-stage constrained equal awards and losses rules for multi-issue allocation situations*
}

\author{
Silvia Lorenzo-Freire ${ }^{1,2} \quad$ Balbina Casas-Méndez ${ }^{3}$ \\ Ruud Hendrickx ${ }^{4}$
}

\begin{abstract}
This paper considers two-stage solutions for multi-issue allocation situations. Characterisations are provided for the two-stage constrained equal awards and constrained equal losses rules, based on the properties of composition and path independence.
\end{abstract}

Key words: multi-issue allocation situations, constrained equal awards, constrained equal losses, two-stage solutions

JEL classification number: $\mathrm{C} 71$

\section{Introduction}

Calleja et al. (2004) introduced multi-issue allocation situations to model bankruptcy-like problems in which the estate is divided not on the basis of a single claim for each agent, but several claims resulting from so-called issues. As a solution to such a multi-issue allocation situation they present two extensions of the run-to-the-bank rule for bankruptcy situations by O'Neill (1982). These extensions deal with the question what the issues should signify for the division of the estate in a sophisticated way, using compensation payments.

One drawback of their approach, however, is that although the compensations form an elegant mechanism to deal with the issues, the rules are difficult to compute. Moreover, the characterisations of the two rules (using O'Neill-like consistency properties) are rather complex.

\footnotetext{
${ }^{1}$ Departamento de Estatística e IO, Universidade de Vigo.

${ }^{2}$ Corresponding author. Postal address: Departamento de Estatística e IO, Facultade de Económicas, Universidade de Vigo, Campus Universitario Lagoas-Marcosende, 36200 Vigo, Spain. E-mail: silvia_lorenzo@uvigo.es.

${ }^{3}$ Departamento de Estatística e IO, Universidade de Santiago de Compostela.

${ }^{4}$ CentER and Department of Econometrics and Operations Research, Tilburg University.

*The authors would like to thank Rodica Branzei for some very useful comments.
} 
González-Alcón et al. (2003) solve this problem by constructing a different extension of the run-to-the-bank rule. The idea behind their two-stage approach is simple: firstly, one should explicitly allocate money to all the issue, and secondly, the amount allocated to each issue should be divided among the players. ${ }^{1}$ They provide a relatively easy characterisation in terms of a property called issue consistency.

This two-stage approach is also taken in Casas-Méndez et al. (2002). They study bankruptcy problems with a priori unions and use the same idea to first allocate money to the unions and then redivide the money within each union.

In this paper, we apply the two-stage idea to the constrained equal awards and constrained equal losses rule. We characterise the two-stage constrained equal awards rule using properties based on equal treatment (cf. O'Neill (1982)), composition (cf. Young (1988)), independence of irrelevant claims (cf. Curiel et al. (1987)). Our characterisation of the two-stage constrained equal losses rule is based on duality and uses properties based on path-independence (cf. Moulin (1987)) and composition of minimum rights (cf. Curiel et al. (1987)). A surprising feature of these characterisations is that the two-stage construction does not follow directly from the properties used, but is a consequence of the strength of the composition and path independence properties.

The outline of the paper is as follows. We present some necessary preliminaries in section 2 and introduce two-stage multi-issue allocation rules. We characterise the two-stage contrained equal awards rule in section 3 and the constrained equal losses rule in section 4 .

\section{Two-stage rules for multi-issue allocation si- tuations}

\section{$2.1 \quad$ Preliminaries}

In this preliminary subsection we give some basic definitions related to multiissue allocation situations that we use through our paper.

A bankruptcy problem (O'Neill (1982)) is a triple $(N, E, c)$ such that $N=$ $\{1, \ldots, n\}$ is the set of players, $E \geq 0$ represents the estate, which is the available amount to satisfy the players' claims, and $c \in \mathbb{R}_{+}^{N}$ is the vector of claims, where

\footnotetext{
${ }^{1}$ In fact, González-Alcón et al. (2003) do not simply apply the bankruptcy run-to-the-bank rule twice, but rather the underlying run-to-the-bank (marginal) vectors and then take the average.
} 
$c_{i}$ denotes the claim of player $i$. The main assumption of a bankruptcy problem is that the estate is not sufficient to satisfy all the claims, $i e, 0 \leq E \leq \sum_{i \in N} c_{i}$.

A bankruptcy rule is a function $\psi$ which associates with every bankruptcy problem $(N, E, c)$ a vector $\psi(N, E, c) \in \mathbb{R}^{N}$ such that

- $0 \leq \psi(N, E, c) \leq c$,

- $\sum_{i \in N} \psi_{i}(N, E, c)=E$.

A multi-issue allocation (MIA) situation (Calleja et al. (2004)) is a 4-tuple $(R, N, E, C)$, where $R=\{1, \ldots, r\}$ and $N=\{1, \ldots, n\}$ are the sets of issues and players, respectively, $E \geq 0$ is the estate to be divided and $C \in \mathbb{R}_{+}^{R \times N}$ is the matrix of claims. An element $c_{k i}$ represents the amount claimed by player $i \in N$ according to the issue $k \in R$. We assume that there is a positive claim in each issue and for each player. Moreover, we assume $0 \leq E \leq \sum_{k \in R i \in N} \sum_{k i}$. Note that a bankruptcy problem is a MIA situation with $|R|=1$. For all $k \in R$, $C_{k}=\left(c_{k i}\right)_{i \in N}$ denotes the vector of claims according to issue $k \in R$.

A multi-issue allocation (MIA) solution $\Psi$ is a function that associates with every MIA situation $(R, N, E, C)$ a matrix $\Psi(R, N, E, C) \in \mathbb{R}^{R \times N}$ satisfying reasonability and efficiency:

- $0 \leq \Psi(R, N, E, C) \leq C$,

- $\sum_{k \in R i \in N} \sum_{k i} \Psi_{k}(R, N, E, C)=E$.

Note that Calleja et al. (2004) and González-Alcón et al. (2003) consider vectorvalued MIA solutions (in $\mathbb{R}^{N}$ ) rather than matrix solutions. We need solutions in $\mathbb{R}^{R \times N}$ in order to properly define composition and path independence, both of which deal with reduced claims matrices. Of course, any matrix outcome gives rise to a vector solution by just adding up all the amounts over the issues.

A bankruptcy situation with a priori unions (Casas-Méndez et al. (2002)) gives rise to a MIA situation, where the issues correspond to the unions. In the associated claims matrix, the issues are disjoint in the sense that each player has a claim on just one issue.

\subsection{Two-stage MIA rules}

In this subsection we define a natural two-stage procedure to define MIA solutions from bankruptcy rules. 
Definition 1 Let $\psi$ be a bankruptcy rule and let $(R, N, E, C)$ be a MIA situation. The two-stage solution $\Psi^{\psi}(R, N, E, C)$ is the MIA solution obtained from the following two-stage procedure.

First stage: Consider the so-called quotient bankruptcy problem $\left(R, E, c^{R}\right)$, where $c^{R}=\left(c_{1}^{R}, \ldots, c_{r}^{R}\right) \in \mathbb{R}^{R}$ denotes the vector of total claims in the issues, $i e, c_{k}^{R}=\sum_{i \in N} c_{k i}$ for all $k \in R$. Divide the amount $E$ among the issues using bankruptcy rule $\psi$. In this way, we obtain $\psi\left(R, E, c^{R}\right) \in \mathbb{R}^{R}$.

Second stage: For each $k \in R$, consider a new bankruptcy problem for the players $\left(N, \psi_{k}\left(R, E, c^{R}\right), C_{k}\right)$ and apply the same bankruptcy rule $\psi$ to this new bankruptcy problem. So, we obtain $\psi\left(N, \psi_{k}\left(R, E, c^{R}\right), C_{k}\right) \in \mathbb{R}^{N}$ for each $k \in R$.

The next figure shows the whole procedure:

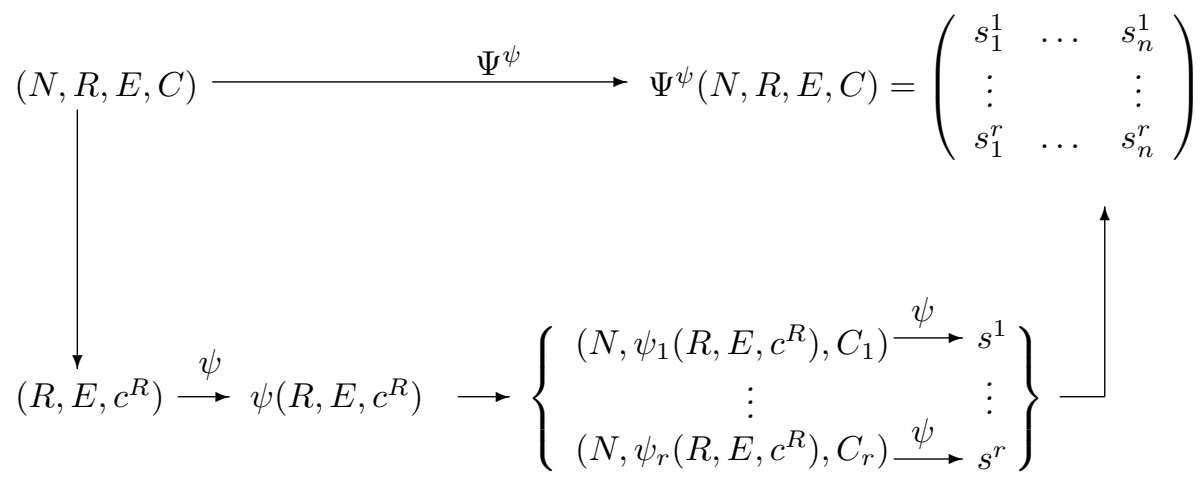

From the definition, it is easy to deduce that each two-stage rule $\Psi^{\psi}$ satisfies consistency in two stages. A rule $\Psi$ satisfies consistency in two stages if for each MIA situation $(R, N, E, C)$ and for all $k \in R, i \in N$,

$$
\Psi_{k i}(R, N, E, C)=\Psi_{k i}\left(\{k\}, N, \Psi_{k 1}\left(R,\{1\}, E, c^{R}\right), C_{k}\right) .
$$

Consistency in two stages is equivalent with the combination of two well-known properties from the bankruptcy literature. The first of these is the quotient property. A rule $\Psi$ satisfies the quotient property if for each MIA situation $(R, N, E, C)$ and for all $k \in R$,

$$
\sum_{i \in N} \Psi_{k i}(R, N, E, C)=\Psi_{k 1}\left(R,\{1\}, E, c^{R}\right) .
$$

This property means that the total quantity assigned to an issue in a MIA situation is equal to the amount assigned to the same issue in the so-called quotient MIA situation, which is the MIA situation with only one player, where the claim of each issue equals the sum of all the players' claims in that issue. 
The second related property is consistency within the issues, which is a direct translation of the (self-)consistency property introduced by Aumann and Maschler (1985). A MIA rule $\Psi$ satisfies consistency within the issues if for each MIA situation $(R, N, E, C)$ and for all $k \in R, i \in N$,

$$
\Psi_{k i}(R, N, E, C)=\Psi_{k i}\left(\{k\}, N, \sum_{j \in N} \Psi_{k j}(R, N, E, C), C_{k}\right) .
$$

This property says that redividing the total amount within an issue using the same rule $\Psi$ should yield the same outcome.

\section{The two-stage constrained equal awards rule}

In this section we provide a characterisation of the two-stage constrained equal awards rule. The constrained equal awards rule for bankruptcy situations $(C E A)$ is defined by $C E A_{i}(N, E, c)=\min \left\{\lambda, c_{i}\right\}$ for all $i \in N$, where $\lambda$ is such that $\sum_{i \in N} \min \left\{\lambda, c_{i}\right\}=E$. The two-stage rule $\Psi^{C E A}$ is then given, for all $(R, N, E, C)$ and for all $k \in R, i \in N$, by

$$
\Psi_{k i}^{C E A}(R, N, E, C)=\min \left\{\beta_{k}, c_{k i}\right\}
$$

where for all $k \in R, \beta_{k}$ is such that $\sum_{i \in N} \min \left\{\beta_{k}, c_{k i}\right\}=\min \left\{\lambda, c_{k}^{R}\right\}$ and $\lambda$ is such that $\sum_{k \in R} \min \left\{\lambda, c_{k}^{R}\right\}=E$.

Note that $\Psi^{C E A}$ differs from another natural extension of the $C E A$ rule, defined by

$$
\Psi_{k i}(R, N, E, C)=\min \left\{\lambda, c_{k i}\right\}
$$

for every $i \in N$ and $k \in R$ with $\lambda$ such that $\sum_{k \in R i \in N} \sum_{i} \min \left\{\lambda, c_{k i}\right\}=E$. A big distinction between the two extensions is that the latter does not satisfy the quotient property.

Below, we list some properties which we will use to characterise the two-stage $C E A$ rule.

Composition. A rule $\Psi$ satisfies composition if for all $(R, N, E, C)$ and for all $0 \leq E^{\prime} \leq E$ we have

$$
\Psi(R, N, E, C)=\Psi\left(R, N, E^{\prime}, C\right)+\Psi\left(R, N, E-E^{\prime}, C-\Psi\left(R, N, E^{\prime}, C\right)\right) .
$$


According to this property, we can divide the total estate among the issues and the players using two different procedures, which result in the same outcome. In the first procedure, we divide the total estate directly using $\Psi$. In the other procedure, we first divide a part $E^{\prime}$ of the estate and then divide remainder $E-E^{\prime}$ on the basis of the remaining claims, both times using $\Psi$.

Independence of irrelevant claims. A rule $\Psi$ satisfies independence of irrelevant claims if for each MIA situation $(R, N, E, C)$ we have

$$
\Psi(R, N, E, C)=\Psi\left(R, N, E, C^{E}\right)
$$

where $C^{E} \in \mathbb{R}_{+}^{R \times N}$ is such that $c_{k i}^{E}=\min \left\{c_{k i}, E\right\}$ for all $k \in R$ and $i \in N$.

This property says that truncating each claim to the estate does not influence the outcome.

Equal treatment for the players within an issue. A rule $\Psi$ satisfies this property if for each MIA situation $(R, N, E, C)$, for all $k \in R$ and $i, j \in$ $N$ such that $c_{k i}=c_{k j}, \Psi_{k i}(R, N, E, C)=\Psi_{k j}(R, N, E, C)$.

Equal treatment for the issues. A rule $\Psi$ satisfies equal treatment for the issues if for all MIA situation $(R, N, E, C)$, for all $k, k^{\prime} \in R$ such that $c_{k}^{R}=$ $c_{k^{\prime}}^{R}, \Psi_{k 1}\left(R,\{1\}, E, c^{R}\right)=\Psi_{k^{\prime} 1}\left(R,\{1\}, E, c^{R}\right)$.

Proposition 1 The two-stage constrained equal awards rule, $\Psi^{C E A}$, satisfies the properties of composition, independence of irrelevant claims, equal treatment for the players within an issue, equal treatment for issues and the quotient property.

Proof: We will only check that $\Psi^{C E A}$ satisfies the properties of composition and independence of irrelevant claims. The remaining properties follow immediately from the definitions. Let $(\mathrm{R}, \mathrm{N}, \mathrm{E}, \mathrm{C})$ be a MIA situation.

a) Composition.

As composition is satisfied by $C E A$ for bankruptcy problems, taking $\left(R,\{1\}, E, c^{R}\right)$ we have that for all $0 \leq E^{\prime} \leq E$ :

$$
\begin{gathered}
\Psi^{C E A}\left(R,\{1\}, E, c^{R}\right)=\Psi^{C E A}\left(R,\{1\}, E^{\prime}, c^{R}\right)+ \\
\Psi^{C E A}\left(R,\{1\}, E-E^{\prime}, c^{R}-\Psi^{C E A}\left(R,\{1\}, E^{\prime}, c^{R}\right)\right) .
\end{gathered}
$$

Let $k \in R$. Again we apply composition with $0 \leq \Psi_{k}^{C E A}\left(R,\{1\}, E^{\prime}, c^{R}\right) \leq$ $\Psi_{k}^{C E A}\left(R,\{1\}, E, c^{R}\right)$ :

$$
\begin{aligned}
& \Psi^{C E A}\left(\{k\}, N, \Psi_{k}^{C E A}\left(R,\{1\}, E, c^{R}\right), C_{k}\right)= \\
& \Psi^{C E A}\left(\{k\}, N, \Psi_{k}^{C E A}\left(R,\{1\}, E^{\prime}, c^{R}\right), C_{k}\right)+
\end{aligned}
$$




$$
+\Psi^{C E A}\left(\{k\}, N, \Psi_{k}^{C E A}\left(R,\{1\}, E, c^{R}\right)-\Psi_{k}^{C E A}\left(R,\{1\}, E^{\prime}, c^{R}\right), C_{k}^{\prime}\right),
$$

where $C_{k}^{\prime}=C_{k}-\Psi^{C E A}\left(\{k\}, N, \Psi_{k}^{C E A}\left(R,\{1\}, E^{\prime}, c^{R}\right), C_{k}\right)$.

Therefore

$$
\begin{gathered}
\Psi^{C E A}\left(\{k\}, N, \Psi_{k}^{C E A}\left(R,\{1\}, E, c^{R}\right), C_{k}\right)= \\
\Psi^{C E A}\left(\{k\}, N, \Psi_{k}^{C E A}\left(R,\{1\}, E^{\prime}, c^{R}\right), C_{k}\right)+ \\
+\Psi^{C E A}\left(\{k\}, N, \Psi_{k}^{C E A}\left(R,\{1\}, E-E^{\prime}, c^{R}-\Psi^{C E A}\left(R,\{1\}, E^{\prime}, c^{R}\right)\right), C_{k}^{\prime}\right),
\end{gathered}
$$

where $C_{k}^{\prime}=C_{k}-\Psi^{C E A}\left(\{k\}, N, \Psi_{k}^{C E A}\left(R,\{1\}, E^{\prime}, c^{R}\right), C_{k}\right)$.

Finally, using the definition of $\Psi^{C E A}$ and gathering the results of all issues in a matrix, we obtain

$$
\begin{gathered}
\Psi^{C E A}(R, N, E, C)=\Psi^{C E A}\left(R, N, E^{\prime}, C\right)+ \\
+\Psi^{C E A}\left(R, N, E-E^{\prime}, C-\Psi^{C E A}\left(R, N, E^{\prime}, C\right)\right) .
\end{gathered}
$$

b)Independence of irrelevant claims.

Using the definition of $\Psi^{C E A}$ and the fact that the $C E A$ rule for bankruptcy problems is independent of irrelevant claims, we get for all $k \in R$ and $i \in N$ $\Psi_{k i}^{C E A}\left(R, N, E, C^{E}\right)=$

$$
\begin{aligned}
& =\Psi_{k i}^{C E A}\left(\{k\}, N, \Psi_{k 1}^{C E A}\left(R,\{1\}, E,\left(c^{E}\right)^{R}\right), C_{k}^{E}\right) \\
& =\Psi_{k i}^{C E A}\left(\{k\}, N, \Psi_{k 1}^{C E A}\left(R,\{1\}, E,\left(\left(c^{E}\right)^{R}\right)^{E}\right), C_{k}^{E}\right) \\
& =\Psi_{k i}^{C E A}\left(\{k\}, N, \Psi_{k 1}^{C E A}\left(R,\{1\}, E,\left(c^{R}\right)^{E}\right), C_{k}^{E}\right) \\
& =\Psi_{k i}^{C E A}\left(\{k\}, N, \Psi_{k 1}^{C E A}\left(R,\{1\}, E, c^{R}\right), C_{k}^{E}\right) \\
& =\Psi_{k i}^{C E A}\left(\{k\}, N, \Psi_{k 1}^{C E A}\left(R,\{1\}, E, c^{R}\right),\left(C_{k}^{E}\right)^{C E A}\left(R,\{1\}, E, c^{R}\right)\right) \\
& =\Psi_{k i}^{C E A}\left(\{k\}, N, \Psi_{k 1}^{C E A}\left(R,\{1\}, E, c^{R}\right), C_{k}^{\Psi_{k 1}^{C E A}\left(R,\{1\}, E, c^{R}\right)}\right) \\
& =\Psi_{k i}^{C E A}\left(\{k\}, N, \Psi_{k 1}^{C E A}\left(R,\{1\}, E, c^{R}\right), C_{k}\right) \\
& =\Psi_{k i}^{C E A}(R, N, E, C) .
\end{aligned}
$$

Theorem 1 There is only one rule which satisfies the properties of composition, independence of irrelevant claims, equal treatment for the players within an issue, equal treatment for issues and quotient property: the two-stage constrained equal awards rule $\Psi^{C E A}$.

Proof: In view of Proposition 1, we only need to prove uniqueness. Let $(R, N, E, C)$ be a MIA situation and let $\Psi$ be a rule satisfying the five properties. We divide the proof into two parts. In part I, we show that $C E A$ should be applied in the quotient problem, ie,

$$
\Psi\left(R,\{1\}, E, c^{R}\right)=\Psi^{C E A}\left(R,\{1\}, E, c^{R}\right) .
$$


In part II, we show that $C E A$ should be used within each issue. Part I follows the proof of Dagan (1996), but is repeated here to facilitate part II.

\section{Part I:}

We consider the corresponding quotient problem $\left(R,\{1\}, E, c^{R}\right)$ and assume, without loss of generality, that $0 \leq c_{1}^{R} \leq c_{2}^{R} \leq \ldots \leq c_{r}^{R}$.

If $E \leq c_{1}^{R}:=E^{1}$, then because of the properties of independence of irrelevant claims and equal treatment for the issues, the estate is split into equal parts and, as a result, (2) holds.

If $E^{1}<E \leq c_{1}^{R}+c_{1}^{R}\left(1-\frac{1}{r}\right):=E^{2}$, then the first case together with composition yields

$$
\begin{aligned}
\Psi\left(R,\{1\}, E, c^{R}\right)= & \Psi^{C E A}\left(R,\{1\}, E^{1}, c^{R}\right)+ \\
& +\Psi\left(R,\{1\}, E-E^{1}, c^{R}-\Psi^{C E A}\left(R,\{1\}, E^{1}, c^{R}\right)\right)
\end{aligned}
$$

and $E-E^{1} \leq c_{1}^{R}\left(1-\frac{1}{r}\right)=c_{1}^{R}-\Psi_{11}^{C E A}\left(R,\{1\}, E^{1}, c^{R}\right)$. Again, because of independence of irrelevant claims and equal treatment for the issues, we divide the amount $E-E^{1}$ into identical parts and hence

$$
\begin{aligned}
& \Psi\left(R,\{1\}, E-E^{1}, c^{R}-\Psi^{C E A}\left(R,\{1\}, E^{1}, c^{R}\right)\right)= \\
= & \Psi^{C E A}\left(R,\{1\}, E-E^{1}, c^{R}-\Psi^{C E A}\left(R,\{1\}, E^{1}, c^{R}\right)\right)
\end{aligned}
$$

and, given that $\Psi^{C E A}$ satisfies composition, (2) holds.

Continuing this procedure with $E^{t}:=E^{t-1}+c_{1}^{R}\left(1-\frac{1}{r}\right)^{t-1}, t \geq 2$, we obtain (2) for all $E<r c_{1}^{R}$. Furthermore, since a rule that satisfies composition is continuous in the estate, the statement also holds for $E=r c_{1}^{R}$.

The next step is to show (2) if $r c_{1}^{R}<E \leq r c_{1}^{R}+(r-1)\left(c_{2}^{R}-c_{1}^{R}\right)$. To show this, we repeat the previous procedure, with $r c_{1}^{R}+\left(c_{2}^{R}-c_{1}^{R}\right)$ taking the role of $E^{1}, r c_{1}^{R}+\left(c_{2}^{R}-c_{1}^{R}\right)+\left(c_{2}^{R}-c_{1}^{R}\right)\left(1-\frac{1}{r-1}\right)$ taking the role of $E^{2}$, and so on. Using composition, we first divide $r c_{1}^{R}$ according to the first step and next we divide the remainder equally among issues $\{2, \ldots, r\}$. We use the same limit argument as in the first step to obtain (2) for all $r c_{1}^{R}<E \leq r c_{1}^{R}+(r-1)\left(c_{2}^{R}-c_{1}^{R}\right)$.

We repeat this procedure, making one issue drop out in each step, until (2) is shown for all possible estates. Then, by the quotient property,

$$
\sum_{i \in N} \Psi_{k i}(R, N, E, C)=\Psi_{k}^{C E A}\left(R,\{1\}, E, c^{R}\right)
$$

for all $k \in R$. 
Part II:

Define $N_{k}=\left\{i \in N: c_{k i}>0\right\}$ and $\rho_{k}=\left|N_{k}\right| \min _{i \in N_{k}} c_{k i}$ for all $k \in R$ and define $\rho=\min _{k \in R} \rho_{k}$. Without loss of generality, assume that claim $c_{11}$ determines this minimum. Then $r \rho$ is the minimum estate to fully sustain claim $c_{11}$ according to $\Psi^{C E A}$. We first show that

$$
\Psi(R, N, E, C)=\Psi^{C E A}(R, N, E, C)
$$

if $E \leq r \rho$ and afterwards show it for $E>r \rho$.

i) In case $E \leq r \rho$, take $E^{1}=\min _{k \in R} \min _{i \in N_{k}} c_{k i}$. Note that this minimum is not necessarily attained by $c_{11}$. First, suppose that it is. If $E \leq E^{1}$, then part $\mathrm{I}$, independence of irrelevant claims and equal treatment for the players within an issue imply (3). Then $\Psi_{k i}(R, N, E, C)=\frac{E}{r\left|N_{k}\right|}$ for all $k \in R, i \in N$. Next, take $E^{2}=E^{1}+\min _{k \in R} \min _{i \in N_{k}}\left(c_{k i}-\Psi_{k i}^{C E A}\left(R, N, E^{1}, C\right)\right)$. If $E^{1}<E \leq E^{2}$, then by composition and the previous step, we have

$$
\begin{aligned}
\Psi(R, N, E, C)= & \Psi^{C E A}\left(R, N, E^{1}, C\right)+ \\
& +\Psi\left(R, N, E-E^{1}, C-\Psi^{C E A}\left(R, N, E^{1}, C\right)\right) .
\end{aligned}
$$

Then

$$
\begin{aligned}
E-E^{1} & \leq \min _{k \in R} \min _{i \in N_{k}}\left(c_{k i}-\Psi_{k i}^{C E A}\left(R, N, E^{1}, C\right)\right) \\
& =c_{11}-\Psi_{11}^{C E A}\left(R, N, E^{1}, C\right)
\end{aligned}
$$

where the equality follows from the fact that the minimum locations for $\rho$ and $E^{1}$ coincide. So, $E^{2}=c_{11}+c_{11}\left(1-\frac{1}{r\left|N_{1}\right|}\right)$. Hence, we can continue the procedure as described in part I to show that (3) holds if $E \leq r\left|N_{1}\right| c_{11}=r \rho$.

Next, suppose that the minimum locations for $\rho$ and $E^{1}$ do not coincide. Then we start with the same procedure as in the previous case. The difference is that now we cannot conclude that in each step, the minimum remaining claim is in the same position. Two things can happen. The easy case occurs if after a finite number of steps the estate is smaller than all remaining claims. In this case, the procedure stops and we have (3) as a consequence of independence of irrelevant claims, part I and equal treatment of the players within an issue.

In the other case, we need to apply a similar limit argument as before. From the construction of $\rho$ it follows that the remaining claim for player 1 in issue 1 is the first to reach zero in our procedure, wherever the minimal remaining claim is located in each step. So, at some stage in the procedure, the minimal remaining 
claim must shift to the (1,1)-position ${ }^{2}$ and stay there. From this point on, we are faced with the easy situation with stationary minimum location as described above and we conclude, using the same limit argument, that (3) holds for all $E \leq r \rho .^{3}$

ii) If $E>r \rho$, we use composition to obtain

$$
\begin{aligned}
\Psi(R, N, E, C)= & \Psi^{C E A}(R, N, r \rho, C)+ \\
& +\Psi\left(R, N, E-r \rho, C-\Psi^{C E A}(R, N, r \rho, C)\right) .
\end{aligned}
$$

Note that in the matrix of remaining claims, there is at least one more zero than in the original claims matrix. For these remaining claims, we again define $N_{k}$ and $\rho_{k}$ for all $k \in R$ and $\rho$ in the same way and we reapply the procedure. The only thing that we have to take care of is that there might be an issue with only zero claims remaining. In this case, this issue is not taken into account when determining $\rho$. When the estate is allocated, the players automatically receive zero in this issue because of reasonability of $\Psi$ and the estate is divided among the issues according to part I.

Hence, in each step, we can apply the procedure to show that (3) holds for ever increasing estates. Since in each step at least one remaining claim becomes zero, we finish in a finite number of steps and conclude that (3) holds for all possible estates.

A nice feature of the characterisation in Theorem 1 is that although the two-stage $C E A$ rule satisfies consistency in two stages, we only need the quotient property and not consistency within the issues. Apparently, composition for MIA solutions is quite a strong generalisation of composition for bankruptcy rules. A more straightforward, but less interesting characterisation could be obtained by including consistency within the issues and weakening composition by replacing it with composition between the issues and composition within the issues.

In the remainder of this section, we show that the properties we have mentioned in Theorem 1 are necessary.

First, the rule defined by (1) satisfies all properties in the theorem except the quotient property.

\footnotetext{
${ }^{2}$ It is possible that it first shifts to other positions, but this does not affect the argument.

${ }^{3}$ In fact, the only difference with the previous situation is that at the start of the procedure, the estate is divided at a different pace. This does not effect the total division in the limit.
} 
Both the two-stage constrained equal losses rule (which we will discuss in the next section) and the two-stage proportional rule (cf. O'Neill (1982)) satisfy the properties of composition, equal treatment for the issues, equal treatment for the players within an issue and the quotient property. Noting that the constrained equal losses rule and the proportional rule for bankruptcy problems satisfy composition and equal treatment, the proof is similar to the proof for the two-stage constrained equal awards rule. However, neither of these rules is independent of irrelevant claims.

On the other hand, the recursive completion rule (cf. O'Neill (1982)) in two stages, the contested garment consistent rule (cf. Aumann and Maschler (1985)) in two stages and the adjusted proportional rule (cf. Curiel et al. (1987)) in two stages satisfy independence of irrelevant claims, equal treatment for the issues, equal treatment for the players within an issue and quotient problem but do not satisfy the property of composition.

Let $(R, N, E, C)$ be a multi-issue allocation situation and $\sigma \in \Pi(N)$ (the set of orders over $N)$. We define a rule using the following procedure. First, we consider the quotient bankruptcy problem $\left(R, E, c^{R}\right)$ and divide the amount $E$ among the issues using the bankruptcy rule $C E A$. In this way, we obtain $C E A_{k}\left(R, E, c^{R}\right)$ for every $k \in R$. Second, for all $k \in R$, consider a new bankruptcy problem for the players, $\left(N, C E A_{k}\left(R, E, c^{R}\right), C_{k}\right)$, and apply the bankruptcy rule $f^{\sigma}$, defined by

$$
f_{i}^{\sigma}(N, E, c)=\min \left\{\max \left\{0, E-\sum_{j: \sigma^{-1}(j)<\sigma^{-1}(i)} c_{j}\right\}, c_{i}\right\}
$$

for every $i \in N$, where $\sigma(p)$ denotes the agent in position $p$ according to the order $\sigma$. In this way, we have that for all $i \in N$ and for all $k \in R$,

$$
\Psi_{k i}(R, N, E, C)=\min \left\{\max \left\{0, \min \left\{\lambda, c_{k}^{R}\right\}-\sum_{j: \sigma^{-1}(j)<\sigma^{-1}(i)} c_{k j}\right\}, c_{k i}\right\}
$$

with $\lambda$ such that $\sum_{k \in R} \min \left\{\lambda, c_{k}^{R}\right\}=E$.

This multi-issue allocation solution satisfies all the properties we have mentioned before, except equal treatment for the players within an issue. We can easily check that this rule satisfies the properties of independence of irrelevant claims, equal treatment for the issues and the quotient property. To prove that this rule satisfies composition, it suffices to show that $f$ satisfies composition in the 
context of bankruptcy problems ${ }^{4}$. Take a bankruptcy problem $(N, E, c)$ and an estate $E^{\prime}$ with $0 \leq E^{\prime} \leq E$. For every $i \in N$, we have that

$$
\begin{gathered}
f_{i}^{\sigma}\left(N, E-E^{\prime}, c-f^{\sigma}\left(N, E^{\prime}, c\right)\right) \\
=\min \left\{\max \left\{0, E-E^{\prime}-\sum_{j: \sigma^{-1}(j)<\sigma^{-1}(i)}\left[c_{j}-f_{j}^{\sigma}\left(N, E^{\prime}, c\right)\right]\right\}, c_{i}-f_{i}^{\sigma}\left(N, E^{\prime}, c\right)\right\}
\end{gathered}
$$

with

$$
f_{i}^{\sigma}\left(N, E^{\prime}, c\right)=\min \left\{\max \left\{0, E^{\prime}-\sum_{j: \sigma^{-1}(j)<\sigma^{-1}(i)} c_{j}\right\}, c_{i}\right\} .
$$

Moreover, we know that

$$
\begin{gathered}
\sum_{j: \sigma^{-1}(j)<\sigma^{-1}(i)} f_{j}^{\sigma}\left(N, E^{\prime}, c\right) \\
=\left\{\begin{array}{cc}
E^{\prime} & \text { if } E^{\prime}-\sum_{j: \sigma^{-1}(j)<\sigma^{-1}(i)} c_{j} \leq 0, \\
\sum_{j: \sigma^{-1}(j)<\sigma^{-1}(i)} c_{j} & \text { if } E^{\prime}-\sum_{j: \sigma^{-1}(j)<\sigma^{-1}(i)}>0,
\end{array}\right.
\end{gathered}
$$

and then,

$$
\begin{gathered}
\max \left\{0, E-E^{\prime}-\sum_{j: \sigma^{-1}(j)<\sigma^{-1}(i)}\left[c_{j}-f_{j}^{\sigma}\left(N, E^{\prime}, c\right)\right]\right\} \\
=\max \left\{0, E-\max \left\{E^{\prime}, \sum_{j: \sigma^{-1}(j)<\sigma^{-1}(i)} c_{j}\right\}\right\} \\
=\max \left\{0, E-\sum_{j: \sigma^{-1}(j)<\sigma^{-1}(i)} c_{j}\right\}-\max \left\{0, E^{\prime}-\sum_{j: \sigma^{-1}(j)<\sigma^{-1}(i)} c_{j}\right\} .
\end{gathered}
$$

Thus,

$$
\begin{aligned}
& f_{i}^{\sigma}\left(N, E-E^{\prime}, c-f^{\sigma}\left(N, E^{\prime}, c\right)\right) \\
= & \min \left\{\max \left\{0, E-\sum_{j: \sigma^{-1}(j)<\sigma^{-1}(i)} c_{j}\right\}\right.
\end{aligned}
$$

${ }^{4} \mathrm{~A}$ similar argument as for $\Psi^{C E A}$ can then be made to show that this MIA solution satisfies composition. 


$$
\begin{gathered}
\left.-\max \left\{0, E^{\prime}-\sum_{j: \sigma^{-1}(j)<\sigma^{-1}(i)} c_{j}\right\}, c_{i}-f_{i}^{\sigma}\left(N, E^{\prime}, c\right)\right\} \\
=\min \left\{\max \left\{0, E-\sum_{j: \sigma^{-1}(j)<\sigma^{-1}(i)} c_{j}\right\}-f_{i}^{\sigma}\left(N, E^{\prime}, c\right), c_{i}-f_{i}^{\sigma}\left(N, E^{\prime}, c\right)\right\} \\
=\min \left\{\max \left\{0, E-\sum_{j: \sigma^{-1}(j)<\sigma^{-1}(i)} c_{j}\right\}, c_{i}\right\}-f_{i}^{\sigma}\left(N, E^{\prime}, c\right) \\
=f_{i}^{\sigma}(N, E, c)-f_{i}^{\sigma}\left(N, E^{\prime}, c\right),
\end{gathered}
$$

where the second equality follows from distinguishing between cases. Hence, we conclude that $f^{\sigma}$ satisfies composition.

Let $(R, N, E, C)$ be a multi-issue allocation situation and let $\tau \in \Pi(R)$. We define a rule using the following procedure. First we consider the bankruptcy problem $\left(R, E, c^{R}\right)$ and divide the amount $E$ among the issues using the bankruptcy rule $f^{\tau}$. In this way, we obtain $f_{k}\left(R, E, c^{R}\right)$ for every $k \in R$. Second, for all $k \in R$, consider the new bankruptcy problem for the players, $\left(N, f_{k}\left(R, E, c^{R}\right), C_{k}\right)$, and apply the bankruptcy rule $C E A$. In this way, we have that for all $i \in N$ and for all $k \in R$,

$$
\Psi_{k i}(R, N, E, C)=\min \left\{\beta_{k}, c_{k i}\right\}
$$

with $\beta_{k}$ such that $\sum_{i \in N} \min \left\{\beta_{k}, c_{k i}\right\}=\min \left\{\max \left\{0, E-\sum_{\ell: \tau^{-1}(\ell)<\tau^{-1}(k)} c_{\ell}^{R}\right\}, c_{k}^{R}\right\}$. This rule satisfies all the properties in Theorem 1 except equal treatment for the issues.

\section{The two-stage constrained equal losses rule}

This section considers the constrained equal losses rule, defined for a bankruptcy problem $(N, E, c)$ by $C E L_{i}(N, E, c)=\max \left\{0, c_{i}-\lambda\right\}$ for all $i \in N$, where $\lambda$ is such that $\sum_{i \in N} \max \left\{0, c_{i}-\lambda\right\}=E$. The two-stage extenstion $\Psi^{C E L}$ is then given, for all $(R, N, E, C)$ and all $k \in R$ and $i \in N$, by

$$
\Psi_{k i}^{C E L}(R, N, E, C)=\max \left\{0, c_{k i}-\beta_{k}\right\}
$$

where for all $k \in R, \beta_{k}$ is such that $\sum_{i \in N} \max \left\{0, c_{k i}-\beta_{k}\right\}=\max \left\{0, c_{k}^{R}-\lambda\right\}$ and $\lambda$ is such that $\sum_{k \in R} \max \left\{0, c_{k}^{R}-\lambda\right\}=E$. 
We now mention some properties for MIA solutions, which we use to characterise the two-stage constrained equal losses rule.

Path independence. A MIA solution $\Psi$ satisfies path independence if for each MIA situation $(R, N, E, C)$ and for all $E^{\prime} \in \mathbb{R}$ such that $E^{\prime} \geq E$,

$$
\Psi(R, N, E, C)=\Psi\left(R, N, E, \Psi\left(R, N, E^{\prime}, C\right)\right) .
$$

If a rule $\Psi$ satisfies path independence, we can divide the estate using two procedures yielding the same result. The first procedure is to divide the money directly using $\Psi$. In the second procedure, we first divide a bigger estate $E^{\prime} \geq E$ and then use the outcome $\Psi\left(N, R, E^{\prime}, C\right)$ as claims matrix to divide the real estate $E$, both times using $\Psi$.

Composition of minimum rights. A MIA solution $\Psi$ satisfies composition of minimum rights if for each MIA situation $(R, N, E, C)$

$$
\begin{gathered}
\Psi(R, N, E, C)=m(R, N, E, C) \\
+\Psi\left(R, N, E-\sum_{k \in R} \sum_{i \in N} m_{k i}(R, N, E, C), C-m(R, N, E, C)\right),
\end{gathered}
$$

where the minimum right of player $i$ in issue $k$ is given by $m_{k i}(R, N, E, C)=$ $\max \left\{0, E-\sum_{\substack{\ell \in R, j \in N \\(\ell, j) \neq(k, i)}} c_{\ell j}\right\}$.

Duality. A MIA solution $\Psi^{*}$ is the dual of another MIA solution $\Psi$ if for each MIA situation $(R, N, E, C)$

$$
\Psi^{*}(R, N, E, C)=C-\Psi\left(R, N, \sum_{k \in R} \sum_{i \in N} c_{k i}-E, C\right) .
$$

This property asserts that one rule is the dual of another one if it assigns to each player in each issue what this player demands minus what this agent obtains dividing the total losses according to the other rule. A rule $\Psi$ is called self-dual if $\Psi^{*}=\Psi$.

The following lemma follows immediately from the observation that both $C E A$ and $C E L$ are dual rules for bankruptcy situations.

Lemma $1\left(\Psi^{C E A}\right)^{*}=\Psi^{C E L}$.

The property $P^{*}$ is the dual property of $P$ if and only if for all MIA solution $\Psi$ that satisfies property $P$ the dual MIA solution $\Psi^{*}$ satisfies property $P^{*}$. The next two lemmas are extensions of results from Herrero and Villar (2001). The proofs are similar. 
Lemma 2 If the MIA solution $\Psi$ is characterised by means of independent properties, then the dual MIA solution $\Psi^{*}$ can be characterised with the dual properties. Moreover, these dual properties are independent.

\section{Lemma 3}

- Composition and path independence are dual properties.

- Independence of irrelevant claims and composition of minimum rights are dual properties.

- The quotient property, equal treatment for the players within an issue and equal treatment in the issues are self-dual.

Using the two previous lemmas and our characterisation of the two-stage constrained equal awards rule, we characterise the constrained equal losses rule in the following theorem.

Theorem 2 There is only one rule which satisfies the properties of path independence, composition of minimum rights, equal treatment for the players within an issue, equal treatment for issues, and the quotient property. This rule is the two-stage constrained equal losses rule $\Psi^{C E L}$.

Similar to the comment following Theorem 1, one can state that path independence for MIA solutions is quite a strong generalisation of path independence for bankruptcy rules.

\section{References}

Aumann, R., and M. Maschler (1985). Game theoretic analysis of a bankruptcy problem from the Talmud. Journal of Economic Theory, 36, 195-213.

Calleja, P., P. Borm, and R. Hendrickx (2004). Multi-issue allocation situations. European Journal of Operational Research, 164, 730-747.

Casas-Méndez, B., P. Borm, L. Carpente, and R. Hendrickx (2002). The constrained equal award rule for bankruptcy situations with a priori unions. CentER Discussion Paper 2002-83, Tilburg University, Tilburg, The Netherlands. (To appear in Annals of Operations Research). 
Curiel, I., M. Maschler, and S. Tijs (1987). Bankruptcy games. Zeitschrift für Operations Research (Now: Mathematical Methods of Operations Research), 31, 143-159.

Dagan, N. (1996). New characterizations of old bankruptcy rules. Social Choice and Welfare, 13, 51-59.

González-Alcón, C., P. Borm, and R. Hendrickx (2003). A composite runto-the-bank rule for multi-issue allocation situations. CentER Discussion Paper 2003-59, Tilburg University, Tilburg, The Netherlands.

Herrero, C., and A. Villar (2001). The three musketeers: four classical solutions to bankruptcy problems. Mathematical Social Sciences, 42, 307-328.

Moulin, H. (1987). Equal or proportional division of a surplus, and other methods. International Journal of Game Theory, 16, 161-186.

O'Neill, B. (1982). A problem of rights arbitration from the Talmud. Mathematical Social Sciences, 2, 345-371.

Young, H. P. (1988). Distributive justice in taxation. Journal of Economic Theory, 44, 321-335. 